Article

\title{
Pulse Compression of Ultrashort UV Pulses by Self-Phase Modulation in Bulk Material
}

\section{Nils Krebs, Igor Pugliesi and Eberhard Riedle *}

Lehrstuhl für BioMolekulare Optik, Ludwig-Maximilians-Universität München (LMU), 80538 München, Germany; E-Mails: nils.krebs@physik.uni-muenchen.de (N.K.); igor.pugliesi@physik.uni-muenchen.de (I.P.)

* Author to whom correspondence should be addressed; E-Mail: riedle @physik.lmu.de; Tel.: +49-89-2180-9210; Fax: +49-89-2180-9202.

Received: 21 December 2012; in revised form: 5 February 2013 / Accepted: 6 February 2013 / Published: 8 February 2013

\begin{abstract}
The bandwidth of ultrafast pulses in the UV is limited by the finite acceptance bandwidth of the nonlinear crystals used for their generation. For fundamental laser pulses it is well established that spectral broadening can be used to overcome intrinsic bandwidth limits. We show that self-phase modulation of UV pulses in bulk materials leads to large spectral broadening and allows for a significant reduction of the pulse duration. We find that for pulse energies in the range of a few $\mu \mathrm{J}$, a thin crystal is favorable due to the strong dispersion in the UV and the limitations set by self-focusing. In contrast to spectral broadening in gaseous media, the self-focus has to lie outside the crystal to avoid beam break up. We focus UV pulses into a $1 \mathrm{~mm}$ thick $\mathrm{CaF}_{2}$ crystal. For moderately short input pulses, a shortening factor up to 2.4 is achieved: the 120 fs long third harmonic output of a Ti:sapphire amplifier is compressed down to $50 \mathrm{fs}$ FWHM. For a central wavelength of $315 \mathrm{~nm}$, we generate pulses as short as $14.9 \mathrm{fs}$ after compression with an UV pulse shaper. In both cases the resulting beam shape is close to Gaussian and fully usable for spectroscopic experiments. We use the pulses in a collinear 2D-UV experiment and clearly resolve vibronic off-diagonal peaks of the $S_{2}{ }^{1} B_{2 u}$ vibronic progression of pyrene.
\end{abstract}

Keywords: ultrashort optical pulses; ultraviolet; UV; self-phase modulation; pulse compression 


\section{Introduction}

For nonlinear optics and time resolved spectroscopic studies of atoms, molecules and biological processes there is a rising need for ultrashort light pulses in the UV and deep UV spectral domain. Straightforward frequency upconversion of short visible pulses in non-linear crystals already allows for the generation of sub-20 fs UV pulses [1] and $30 \mathrm{fs}$ pulses down to $189 \mathrm{~nm}$ [2]. The phase-matching bandwidth can be increased by advanced angular dispersion methods leading to sub-10 fs pulses $[3,4]$. Other important techniques capable of generating UV pulses are four wave mixing in a gas filled hollow wave guide providing sub-10 fs pulses at $270 \mathrm{~nm}$ [5]. Third harmonic generation of the fundamental pulses at around $800 \mathrm{~nm}$ provide sub-30 fs pulses in air [6] or even 3-fs pulses in a Neon filled gas cell [7]. These techniques are characterized by a low conversion efficiency and require an elaborate experimental implementation.

Pulse shortening can also be achieved through spectral broadening of longer UV pulses typically generated by frequency up conversion. Spectral broadening can be achieved by nonlinear phase modulation of the UV pulse in an optically transparent medium. In cross phase modulation the nonlinearity is induced by an auxiliary high intensity laser pulse. To increase the interaction length, gas filled hollow waveguides are often used. With this approach cross phase modulation between the fundamental and the third harmonic of a Ti:sapphire laser amplifier allows to compress the $266 \mathrm{~nm}$ pulses by a factor of 2 down to 23 fs [8]. For a 35 fs Ti:sapphire laser system this process helps to further broaden the intense $266 \mathrm{~nm}$ pulses originating from four-wave-mixing of blue and red pulses down to a length of 8 -fs [9].

Efficient spectral pulse broadening in gas-filled hollow fibers or gas cells has also been achieved by self-phase modulation (SPM). With input pulse lengths of around $100 \mathrm{fs}$, deep UV pulses with an pulse energy of several hundred $\mu \mathrm{J}$ as short as $20 \mathrm{fs}$ centered at $268 \mathrm{~nm}$ [10] and $25 \mathrm{fs}$ centered at $248 \mathrm{~nm}$ [11] were obtained. This technique however needs high input UV pulse energies up to several hundred $\mu \mathrm{J}$ due to the low nonlinearity of the gas. Such high energy pulses are typically not available in spectroscopic setups, particularly at high repetition rates, and therefore the practical use of spectral broadening in gases is limited.

Bulk materials and fibers in contrast offer medium to high nonlinearities and are suitable candidates for nonlinear phase modulation. In single-mode fibers the pulse power is typically limited to tens of $\mathrm{nJ}$. Fibers are therefore best suited for high repetition laser systems [12]. Bulk materials can be operated at any input energy as the thickness of the material can be suitably adjusted so that self-focusing in the crystal is controlled and any serious beam distortion or material destruction can be avoided. Spectral broadening by SPM in bulk material has been first observed in the visible by Alfano et al. [13] and later in the UV by Hata et al. [14]. Theoretically, it was shown that in bulk material cross phase modulation should be capable of generating chirp free nearly-single cycle pulses [15]. The shortening of IR and VIS pulses by SPM in bulk materials has been realized successfully in the 1980s [16,17] and it was envisioned that this approach can be extended into the UV spectral domain [17].

In this paper we demonstrate that SPM can indeed be used to shorten UV pulses below their input Fourier limit. Taking into account the nonlinear processes of self-focusing and SPM we use simulations to find the optimal conditions for UV spectral broadening in common crystals. We then describe our experimental results on spectral broadening of UV pulses in $\mathrm{CaF}_{2}$ plate and describe the 
way to achieve shorter pulses by incorporating a few supplemental parts into the setup. Compression of the pulses to nearly their Fourier limit is demonstrated by implementing an acousto-optical pulse shaper not necessary if the residual higher order chirp is of no concern.

Finally we apply the broadened UV pulses as pump and probe in a collinear two-dimensional experiment that allows us to resolve the spectral correlations of two vibrational bands of pyrene due to the enlarged spectral window of the pulses.

\section{Parameter Optimization for Spectral Broadening}

SPM modifies the spectral content of a laser pulse. The result of this modification is influenced by several experimental factors such as the pulse intensity, the wavelength and the nonlinear material properties. When an intense laser pulse propagates through a medium it induces a dynamic refractive index change $n(t)=n_{0}+n_{2} I(t)$ due to the third-order Kerr nonlinearity $n_{2}$. This leads to self-focusing, self-steepening and SPM. In the case of SPM, $n_{2}$ causes a change of the phase of the electric field in the pulse itself. For an input pulse with spatial and temporal intensity profile $I(r, t)$, this nonlinear phase variation accumulates over the propagation direction $z$ in a medium with thickness $l$ according to the $B$ integral:

$$
B(r, t)=\frac{\omega_{0}}{c} \int_{0}^{l} n_{2} \cdot I(z, r, t) d z
$$

Here $\omega_{0}$ is the initial circular frequency, $c$ the speed of light and $r$ parameterizes the spatial intensity distribution perpendicular to the propagation direction. The accumulated phase shift results in a corresponding frequency shift and thus a broadening or narrowing of the pulse spectrum.

A quantification of the maximum frequency shift due to SPM is given by

$$
\Delta \omega_{\max }=\omega(t)-\omega_{0}=-\left(\frac{\partial}{\partial t} B(t)\right)_{\max }
$$

where the maximum frequency shift $\Delta \omega_{\max }$ is typically located at the maximum slope of the leading and trailing edge of the pulse intensity.

From Equations (1) and (2) one can conclude that high intensities and a long propagation distance in a material with a large positive $n_{2}$ is favorable to maximize spectral broadening through SPM. However, in bulk materials the large dispersion experienced by a UV pulse has to be taken into account. This leads to a reduction of the pulse intensity and thus the accumulated phase. A further limiting factor is the nonlinear process of self-focusing. As often mentioned in the literature, if the self-focus lies within the crystal length, severe beam distortions, beam breakup [14] or ultimately filamentation occurs $[10,18]$.

To illustrate the influence of the material's dispersion on SPM we calculated the $B$ integral (Equation (1)) for different thicknesses of $\mathrm{a} \mathrm{CaF}_{2}$ crystal $\left(n_{2}=1.92 \times 10^{-16} \mathrm{~cm}^{2} / \mathrm{W}\right.$ [19]). The results for a $25 \mathrm{fs}$ long UV pulse centered at $308 \mathrm{~nm}$ with a pulse energy of $3 \mu \mathrm{J}$ are shown in Figure 1 . One should note, that for a given crystal thickness we optimize the beam focusing to a minimal beam waist radius $w$ for which the self-focal length is 1.25 times the crystal length (see orange line in Figure 1). This puts the self-focus outside the crystal and ensures that beam distortions do not occur. The 
self-focal length for a pulse with a power $P$ focused to a beam waist $w$ can be calculated according to Equation (3).

$$
z_{S F}(w)=\frac{0.183 \rho_{0}(w)}{\sqrt{\left(\sqrt{P / P_{c r}\left(n_{2}\right)}-0.852\right)^{2}-0.0219}}
$$

with $\rho_{0}$ being the Rayleigh length and $P_{c r}$ the critical self-focusing power threshold [20,21]. In the simulations, the change in the peak intensity due to self-focusing and self-steepening is neglected. The spatial intensity distribution is assumed to be Gaussian and the input pulses are Fourier limited. For the calculation of the $B$ integral we integrate over the whole beam profile.

Figure 1. Amount of self-phase modulation (SPM) for $25 \mathrm{fs}, 308 \mathrm{~nm}, 3 \mu \mathrm{J}$ UV pulses broadened in $\mathrm{CaF}_{2}$ crystal. Calculated $\mathrm{B}$-integral at increasing levels of theory for varied crystal length. The input beam waist is set to a value for which the self-focal length is $1.25 \times$ the crystal length.

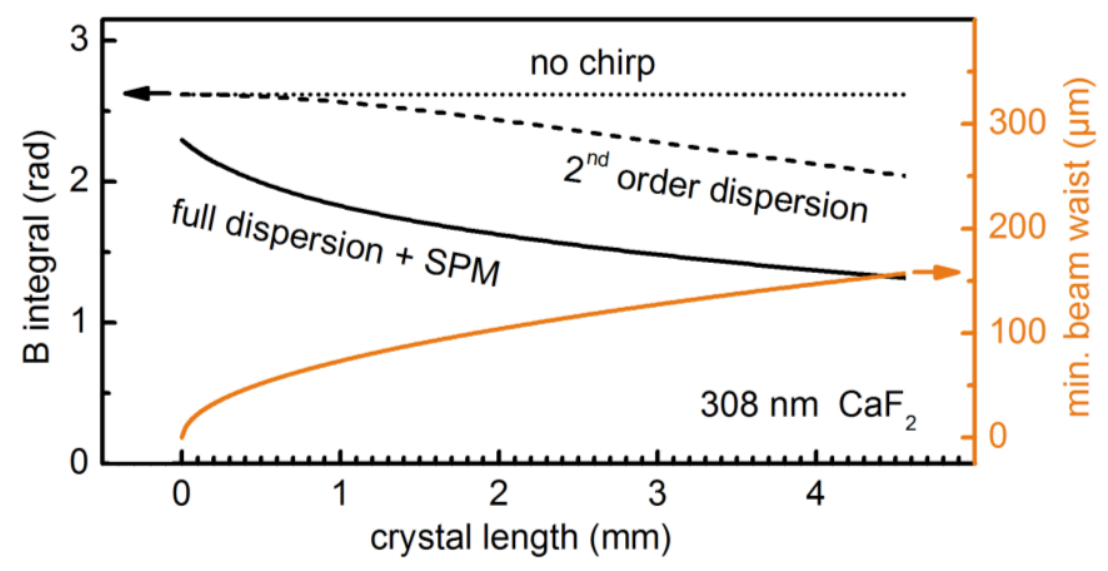

The B integral does not vary with the crystal length when no material dispersion is included in the simulation (Figure 1, dotted line) as the self-focal length is kept 1.25 times the crystal length for the different crystal thicknesses. As soon as 2 nd order dispersion is considered (for a formula see, e.g., [20]), the $B$ integral starts dropping steadily when the crystal thickness exceeds $1 \mathrm{~mm}$ and converges to zero for long crystals (Figure 1, dashed line). The effect is even more dramatic when the full material dispersion is introduced by using virtual femtosecond laboratory software Lab2 [22]. The resulting $B$ integral (Figure 1, continuous line) shows a pronounced decrease even for crystals thinner than a few $\mathrm{mm}$.

To find the optimal experimental conditions for a specific crystal thickness and a given minimal beam waist, we varied the input pulse energies and the type of bulk materials (Figure 2). To get direct information on the achieved broadening we calculated the compressibility factor, which is the Fourier limit of the broadened pulse spectrum after propagating through the material divided by the initial Fourier limited pulse length (25 fs for all simulations).

Figure 2 a shows, that for a fixed crystal length an increase of the input pulse energy increases the achievable compressibility factor. This behavior can be understood from Equations (1) and (3). The self-focal length scales roughly inversely with the square root of the pulse power $P$, while the pulse 
intensity is directly proportional to the power. Therefore, the compressibility factor can be estimated to roughly scale with $\sqrt{P}$.

Figure 2. Compressibility factor (ratio between input and output Fourier limit) for a $25 \mathrm{fs}$ pulse. Results for varied input energies, pulses centered at $308 \mathrm{~nm}$, and a $\mathrm{CaF}_{2}$ crystal (a) and for different crystals with $266 \mathrm{~nm}$ pulses of $3 \mu \mathrm{J}$ energy (b). The input beam waist is set to a value for which the calculated self-focal length is $1.25 \times$ the crystal length.

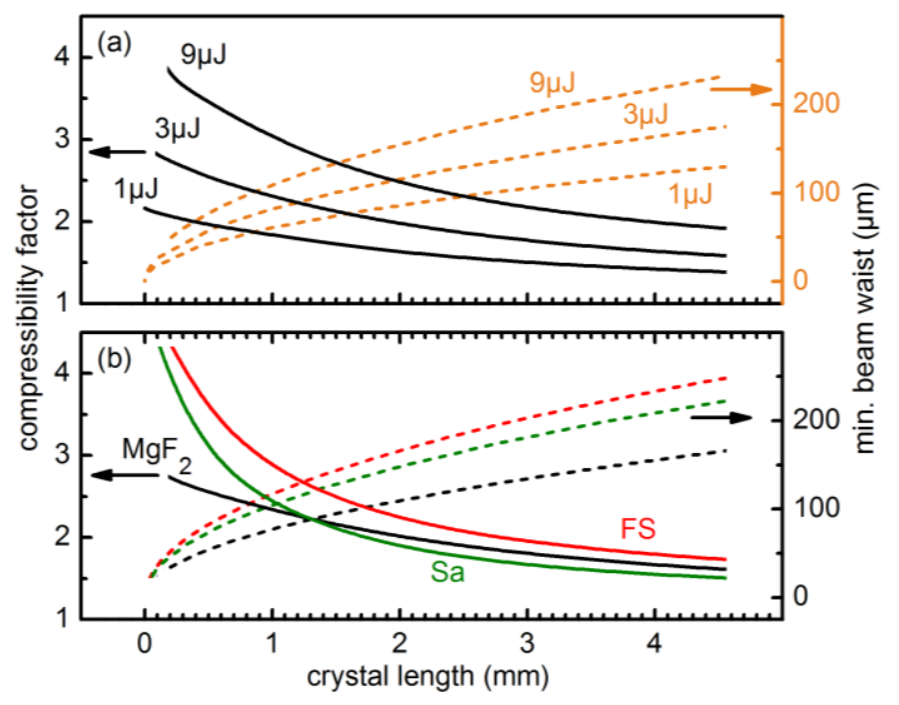

In Figure $2 \mathrm{~b}$ the simulation results for different materials are shown. The $n_{2}$ values of $\mathrm{MgF}_{2}$ $\left(1.5 \times 10^{-16} \mathrm{~cm}^{2} / \mathrm{W}\right)$, fused silica $\left(7.8 \times 10^{-16} \mathrm{~cm}^{2} / \mathrm{W}\right)$ and Sapphire $\left(6.0 \times 10^{-16} \mathrm{~cm}^{2} / \mathrm{W}\right)$ at $266 \mathrm{~nm}$ were taken from the literature [19,23]. The simulations show that a larger $n_{2}$ generally leads to an increase of the compressibility factor. However, the differing dispersion of the materials can change this general trend (see crossing of $\mathrm{MgF}_{2}$ and Sa curves in Figure 2b).

The simulations show that due to the pronounced dispersion in the UV, it is favorable to use short crystals with tight focusing. This is unlike spectral broadening in gases, where meter-long propagations are needed due to the much lower $n_{2}$ values. $1 \mathrm{~mm}$ thick crystals lead to compressibility factors between 2 to 2.5 for Fourier limited Gaussian pulses with energies of a few $\mu \mathrm{J}$. The required beam waist radius lies in the order of $100 \mu \mathrm{m}$. These parameters are experimentally sensible.

\section{Experimental Setup}

A schematic of the setup, which comprises the generation, broadening and characterization of UV pulses, is shown in Figure 3. Tunable visible pulses were generated from a single-stage noncollinear parametric amplifier (NOPA) pumped by a 1-kHz Ti:Sa amplifier system (CPA 2001; Clark MXR) and optimally compressed by a pair of fused silica Brewster prisms. UV pulses with a typical pulse length of $25 \mathrm{fs}$ were generated by frequency doubling or sum frequency mixing the NOPA pulses with the CPA fundamental in BBO crystals with a thickness of $53 \mu \mathrm{m}$. For the frequency doubling we typically obtain $<1 \mu \mathrm{J}$ which corresponds to an efficiency of $<15 \%$. For the sum frequency mixing both beams were collinearly overlapped and the CPA fundamental was focused with a fused silica lens to match the focal size of the beam of the visible pulse in the BBO. Typical energies of $4 \mu \mathrm{J}$ are achieved with a visible to UV efficiency of $50 \%$. 
To introduce a variable positive chirp the UV pulses pass through a set of UV antireflection coated movable fused silica wedges (apex angel $45^{\circ}$ ) with counter oriented apexes [24]. To obtain a clean and spatially symmetric beam profile, which is advantageous for SPM, we introduce an aperture accepting a minimal loss of energy. The UV beam is then focused with the $\mathrm{f}=150 \mathrm{~mm}$ antireflection coated fused silica lens L1 into a few mm thick crystal which spectrally broadens the pulses due to SPM. The position of the crystal relative to the soft focus of the UV beam can be adjusted to obtain maximum broadening without impairing the spatial mode due to self-focusing inside the crystal. A second fused silica lens L2 ( $f=100 \mathrm{~mm}$ ), that can be translated along the beam axis, recollimates the spectrally broadened UV pulse. This not only accounts for the change in divergence caused by the partial self-focusing in the crystal but also adjusts the collimation of the beam such that the specific requirements for pulse shaping with an acousto-optic programmable dispersive filter (AOPDF) are met [1]. Specifically, the intermediate beam waist of the broadened UV pulse after the AOPDF has to be located one focusing length in front of the focusing element which is used to focus the beam into the interaction region, e.g., the sample.

Figure 3. Setup for the generation, compression and characterization of UV pulses spectrally broadened by SPM. PC: prism compressor; SFM (SHG): sum frequency (second harmonic) generation; FS-W: Fused silica wedges; Ap: aperture; L1, L2 lenses.

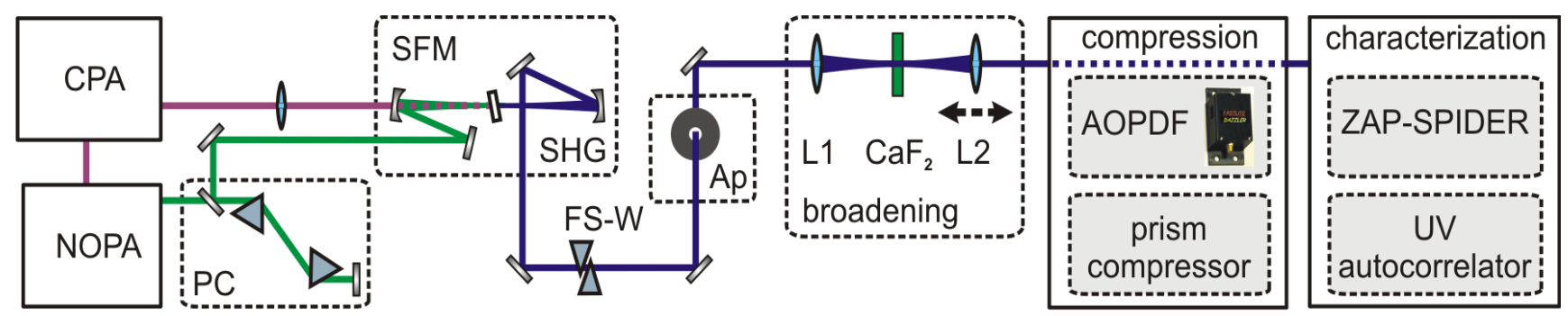

The compression of the positively chirped broadened UV pulses is achieved by either a second UV prism compressor or a combination of a UV prism compressor and an AOPDF (DAZZLER ${ }^{\mathrm{TM}}$ model T-UV-250-400; Fastlite) [25] for higher order chirp correction. The prisms are UV antireflection coated and have an apex angle of $45^{\circ}$. The throughput of the UV PC is roughly $93 \%$. In the PC-AOPDF combination the precompression with the UV PC permits a larger bandwidth of the AOPDF because the acoustic wave is less chirped and thus less elongated within the shaper crystal.

To measure the pulse lengths obtained in the simple setup where only a UV-PC is used to compress the broadened pulses, we employ a newly developed UV autocorrelator [26] based on two photon absorption in a $173 \mu \mathrm{m}$ thick BBO crystal. To check if the higher order chirp contributions of the UV-broadened pulses can be compensated for by the PC-AOPDF combination, we characterized the spectral phase with a ZAP-SPIDER [27].

\section{Results and Discussion}

\subsection{Experimental Characterization of Self-Phase Modulation Broadened UV Pulses}

With the insights gained by the simulations, we experimentally tested the broadening of UV pulses by focusing them into a $\mathrm{CaF}_{2}$ crystal and subsequently compensate the phase of the broadened spectra. 
First we tuned the chirp of the input pulse and investigated the influence of the peak intensity on the SPM process. Subsequently, we varied the type and thickness of the broadening crystal.

When the UV beam is tightly focused into a crystal we observe changes in the spectral intensity distribution (see Figure 4d-f). Experimental conditions can be found that allow the generation of new frequency components due to SPM.

Figure 4. Correlation between the peak intensity and the measured output energy (a), output Fourier limit (b) and the calculated self-focal length (c) obtained by moving a $1 \mathrm{~mm}$ $\mathrm{CaF}_{2}$ crystal relative to the beam focus of a positively chirped pulse centered at $312 \mathrm{~nm}$. Measured spectra and calculated Fourier limits of the input pulse (d) and output pulses for selected crystal positions (e, f).

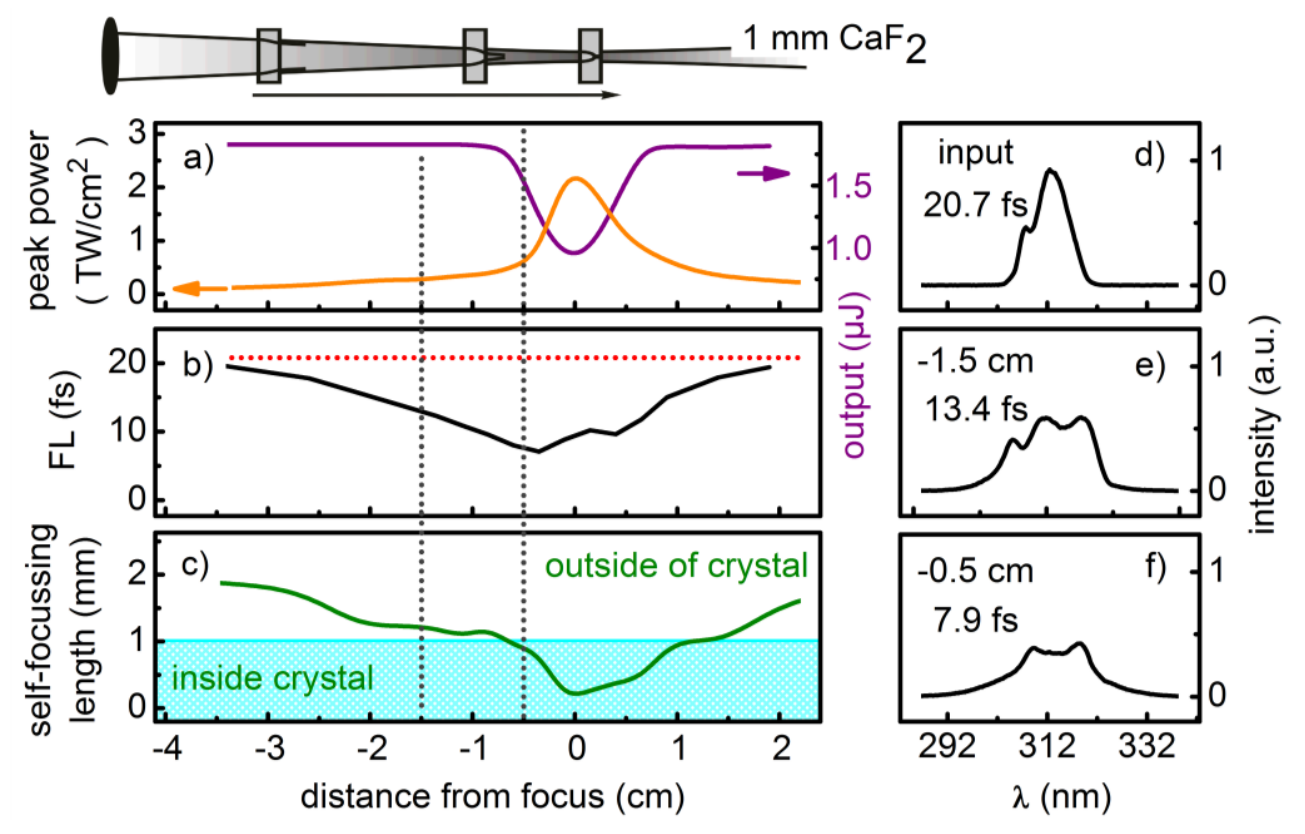

As commonly known for SPM of ultrashort laser pulses, we find that for a negatively chirped input pulse spectral narrowing occurs. For positively chirped pulses - the red components arrive before the blue ones - the output spectra are broadened [28]. Furthermore, for a specific positive chirp (measured to be about $260 \mathrm{fs}^{2}$ ) spectra with a square like shape which are symmetric about the central frequency can be generated. Pulses with such spectra correspond to the maximal broadening observed in the experiment while the beam profile still has a homogeneous spatial intensity distribution. These characteristics are advantageous for applications like pump-probe spectroscopy.

A simple way to vary and optimize the beam waist for a given crystal length and type and thus precisely adjust the peak intensity while using the full pulse energy is to move the crystal with respect to the focus of the UV beam. For this experiment we chose a $1 \mathrm{~mm} \mathrm{CaF}_{2}$ crystal which we move through the focus of a positively chirped pulse centered at $312 \mathrm{~nm}$ with a pulse energy of $1.96 \mu \mathrm{J}$. The pulse length of the input pulse was measured by the UV-AC and found to be $63 \mathrm{fs}$. To determine the peak intensity at the entrance of the crystal we measured the size of the beam profile at the respective crystal positions with a CCD camera.

When moving the crystal towards the focus, the output energy remains constant and is equal to the input energy minus the losses due to reflection. A drop in the output energy occurs for crystal positions 
within $\pm 1 \mathrm{~cm}$ of the focus. This resembles the profile of a $z$-scan and is therefore attributed to multi-photon absorption (MPA) (Figure 4a, purple line). For every position of the crystal we measure the spectrum and calculated the Fourier limit of the output pulse (Figure 4b). The Fourier limit steadily decreases from 20.6 fs (input spectrum) down to $8 \mathrm{fs}$ when the crystal is placed near the focus. In addition to the above measurements the self-focal length was determined for all respective crystal positions (see Figure 4c). The calculations were done by taking into account the convergence or divergence caused by the focusing of the beam according to ref. [29]. For crystal positions within $\pm 1 \mathrm{~cm}$ of the focus we obtain a self-focal length smaller than the crystal thickness.

When comparing the three plots in Figure $4 \mathrm{a}-\mathrm{c}$, it becomes clear, that the MPA induced drop in output power accompanied by increased shot-to-shot fluctuations occurs when the self-focus lies within the crystal. Although in this regime the Fourier limit is well below $10 \mathrm{fs}$ (Figure 4f), the beam undergoes beam breakup [14] accompanied with a strong divergence after the crystal. Furthermore, it shows enhanced fluctuations both in the spatial and spectral intensity distribution. As can be seen from Figure $4 \mathrm{~b}$, putting the self-focus just outside the crystal still delivers a sizable spectral broadening down to a Fourier limit of 13.4 fs (Figure $4 \mathrm{e}, 1.5 \mathrm{~cm}$ in front of the beam focus) without undesirable beam distortions and no loss in output energy. This regime delivers broadened pulses feasible for further applications and justifies the focusing assumptions made in our previous simulations. The resulting change in beam divergence due to the moderate self-focusing is then compensated for by the recollimation lens $\mathrm{L} 2$.

We investigated the spectral broadening by SPM in Sapphire, $\mathrm{CaF}_{2}, \mathrm{LiF}_{2}$ and fused silica crystals of various thicknesses between $0.5-4 \mathrm{~mm}$. For each crystal we work in the regime with optimum SPM broadening as discussed before. In accordance with our theoretical simulations we find that crystals with a higher $n_{2}$ lead to broader pulses. With the smallest $n_{2}, \mathrm{LiF}_{2}$ delivers the smallest broadening. Although sapphire has a larger $n_{2}$ than $\mathrm{CaF}_{2}$, experimentally we observe slightly better broadening in $\mathrm{CaF}_{2}$. Fused silica gives similar results to $\mathrm{CaF}_{2}$. However impurities cause large beam scattering.

When trying different crystal thicknesses, we achieve larger broadening with thinner crystals as predicted by the theory. Best results are obtained for a $0.5 \mathrm{~mm} \mathrm{CaF}_{2}$ plate with a compressibility factor of $\sim 2$. However, the required tight focusing leads to unwanted multi-photon absorption and to beam distortions caused by the progressive deterioration of the crystal. Therefore we employ 1 and $2 \mathrm{~mm}$ thick $\mathrm{CaF}_{2}$ disks for the compression experiments discussed below.

The experimentally achieved spectral broadening is generally about $25 \%$ less than the calculated values in section 2 . We believe that this is due to the chirp of the pulses neglected in the calculations as well as the deviations of the beam profile from a Gaussian like intensity distribution, which lead to significant spatial distortions well before the self-focus length reaches the crystal length.

\subsection{Compression of Self-Phase Modulated UV Pulses}

The availability of sub-25 fs UV pulses generated by parametric amplification and conversion is in general limited in optic laboratories. More often the third harmonic of the Ti:sapphire system is used directly. To show that SPM can be used in such a configuration we employ the third harmonic of our CPA laser as the initial pulse. Pulses centered at $257 \mathrm{~nm}$ with an energy of about $1.5 \mu \mathrm{J}$ are spectrally broadened in a $1 \mathrm{~mm}$ thick $\mathrm{CaF}_{2}$ crystal (Figure 5). The pulses are subsequently compressed by a UV-PC and then measured with the UV autocorrelator. When the full spatial beam profile is used, we 
obtain a reduction of the initial Fourier limit of 80 fs down to 49 fs (Figure 5a). The measured autocorrelation trace (Figure 5b) shows that the Gaussian shape of the input pulse is conserved. The measured pulse length FWHM changes from 120 fs to 73 fs. When only the central part of the broadened pulses is selected by an aperture (pulse energy reduced by 30\%), we obtain a much broader spectrum, as the spatial intensity is higher in the central part. The initial Fourier limit of 80 fs is shortened by nearly a factor of 3 down to $29 \mathrm{fs}$ (Figure 5c). The measured pulse length is 50 fs (Figure 5d). The autocorrelation trace of the broadened pulses shows deviations from a Gaussian shape due to higher order dispersion which could be eliminated by a more sophisticated compression scheme.

Figure 5. Pulse compression of the 120 fs third harmonic of the CPA by SPM broadening in a $1 \mathrm{~mm} \mathrm{CaF}_{2}$ crystal and chirp compensation by a prism compressor. Dotted lines: input pulses; solid lines: spectrally broadened pulses. Spectra $(\mathbf{a}, \mathbf{c})$ and autocorrelation measurements $(\mathbf{b}, \mathbf{d})$ of the complete beam profile (blue lines) and the central part of the beam profile (red lines).

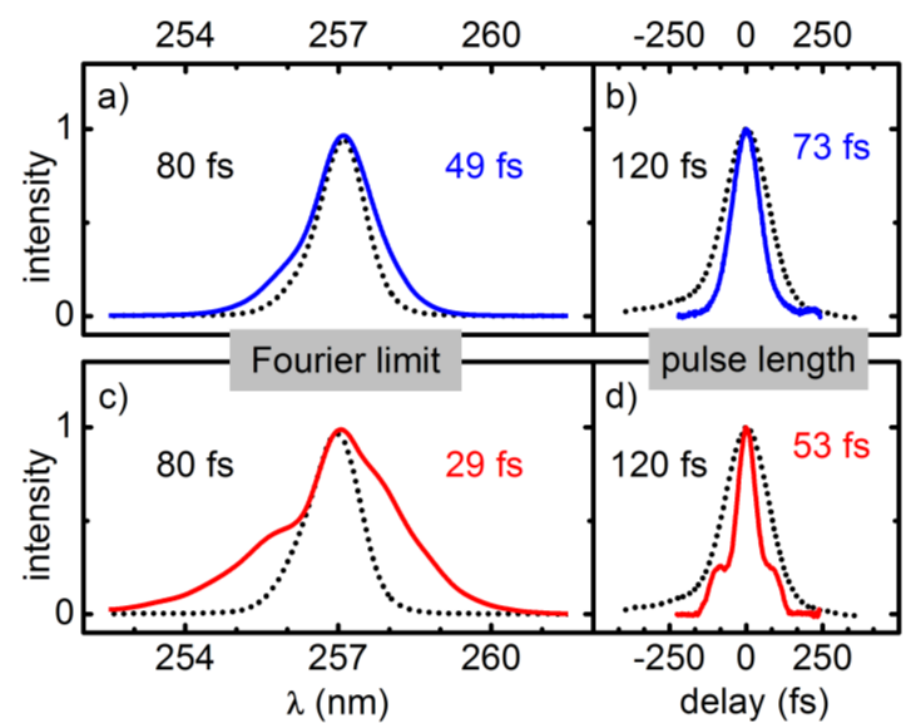

To show that the spectrally broadened pulses are compressible near to the Fourier limit, we employed an UV-PC in combination with an AOPDF to compensate for all orders of dispersion. We compressed SPM broadened pulses centered at $273 \mathrm{~nm}$, generated by frequency doubling the output of the NOPA. Due to the low input energy of $0.7 \mu \mathrm{J}$, we used a $2 \mathrm{~mm}$ thick $\mathrm{CaF}_{2}$ crystal for broadening. The recollimation lens L2 was moved in order to compensate for the SPM induced change in beam divergence while the specific collimation requirements of the AOPDF are met (see ref. [1] for details). According to the literature [17], for a Gaussian beam profile SPM can lead to different frequency chirps across the transverse beam profile related to the spatial intensity profile. Therefore an aperture was used to block the outer parts of the beam. This reduced the pulse energy by about $20 \%$. In order to compensate the chirp of the pulse, the spectral phase was measured by a ZAP-SPIDER and the obtained phase function was then recursively used in the AOPDF to completely flatten the phase. The results are shown in Figure $6 \mathrm{a}$ and $\mathrm{b}$ together with the temporal profile. We are able to obtain a flat spectral phase and the pulse length is reduced to $19.8 \mathrm{fs}$, which is close to the Fourier limit of 19 fs. 
Figure 6. Pulse compression of sub-30 fs UV pulses generated by SHG (a, b) and SFG (c, d) of visible noncollinear parametric amplifier (NOPA) pulses by SPM broadening in a $2 \mathrm{~mm}(\mathbf{a}, \mathbf{b})$ or $1 \mathrm{~mm}(\mathbf{c}, \mathbf{d}) \mathrm{CaF}_{2}$ crystal. Dotted lines: input pulses; solid lines: spectrally broadened pulses. Compression to a flat spectral and temporal phase (dashed lines) was achieved by the use of an acousto-optic pulse shaper and measured by a ZAP-SPIDER.

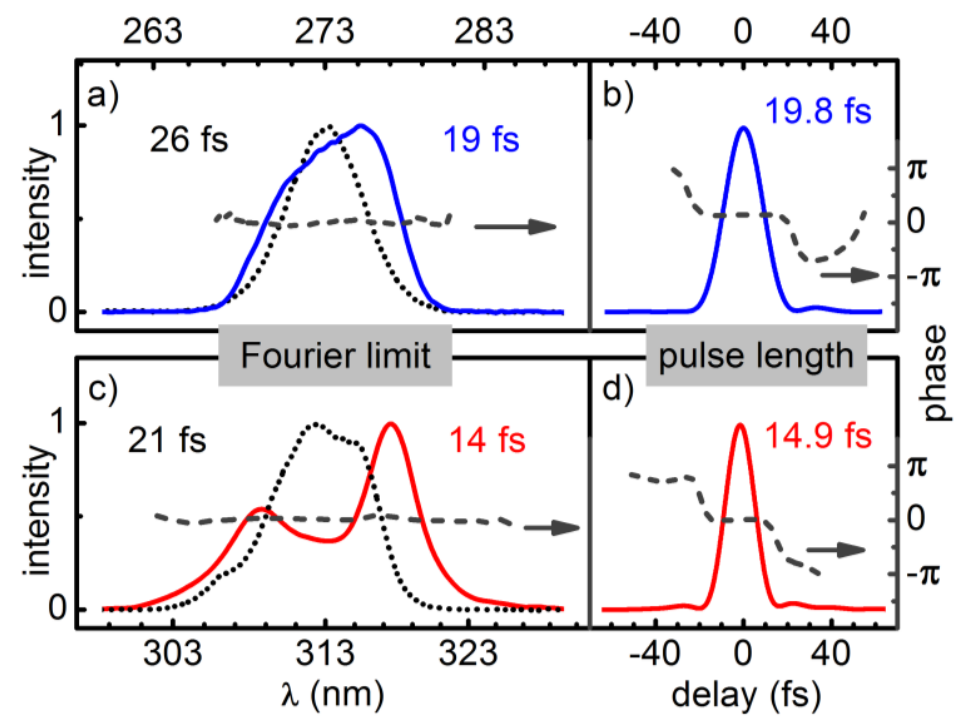

For the pulses with a central wavelength at $313 \mathrm{~nm}$ generated by sum frequency mixing the thickness of $\mathrm{CaF}_{2}$ disk was changed to $1 \mathrm{~mm}$ as more energy $(2.1 \mu \mathrm{J})$ was available. The broadened pulses can again be compressed well below the input Fourier limit to 14.9 fs. This corresponds to a reduction of the pulse length by a factor of about 1.5 of the initial 22 fs long pulses. The energy after the PC was typically about $2 \mu \mathrm{J}$ and that of the compressed pulses after the shaper was about $200 \mathrm{~nJ}$, which corresponds to the $10 \%$ overall efficiency of the shaper device. This is an order of magnitude higher compared to the output energies obtained previously with the AOPDF [1].

\subsection{Effects of Self-Phase Modulation on the Beam Profile}

To investigate the influence of SPM on the beam profile, we use the nearly perfect Gaussian shaped $\left(\mathrm{M}^{2} \sim 1.05\right)$ third harmonic of a Yb:KYW laser (JenLas D2.fs JenOptik GmbH) as input. Pulses with an energy of $310 \mathrm{~nJ}$ and a duration of $173 \mathrm{fs}$ are focused with a fused silica lens ( $\mathrm{f}=50 \mathrm{~mm}$ ) into a $3 \mathrm{~mm}$ thick Sapphire disk. In analogy to the experiments in section 4, the disk is moved relative to the focus along the beam propagation to change the peak intensity and thus the amount of SPM. The beam profile is measured after a second recollimation lens.

When the disk is placed in the focus, we observe strong spectral broadening but severe beam profile distortions as the self-focus lies inside the crystal (see Figure 7b,f). The ring pattern is caused by the interference of spatial variations of self-phase modulation, as explained in refs. [30,31]. Moving the crystal away from the focus significantly reduces the distortions while the spectral broadening is still respectable. Already $2 \mathrm{~mm}$ after the focus a symmetric beam profile is obtained with a broadening that reduces the Fourier limit from 133 fs to 31 fs (see Figure 7d,h). The outer rings in the beam profile can be cut off with an aperture and the inner part then has a beam profile that is comparable with a 
Gaussian beam. The analysis of the measured intensity profile (pink rings in Figure 7a-d) shows that this typically reduces the usable pulse energy to $30 \%$.

Figure 7. Effect of SPM broadening on the beam profile and spectrum of the third harmonic $(342 \mathrm{~nm})$ of the Yb:KYW JenLas laser for various peak powers in a sapphire crystal. The Gaussian shaped $\left(\mathrm{M}^{2} \sim 1.05\right)$ input profile and spectrum are shown in (a) and (e). The crystal was placed in the focus $(\mathbf{b}, \mathbf{f}), 1 \mathrm{~mm}(\mathbf{c}, \mathbf{g})$ and $2 \mathrm{~mm}(\mathbf{d}, \mathbf{h})$ behind the focus. Pink circles indicate the beam energy for various apertures given in percent of the total energy. Fourier limits are given in blue.
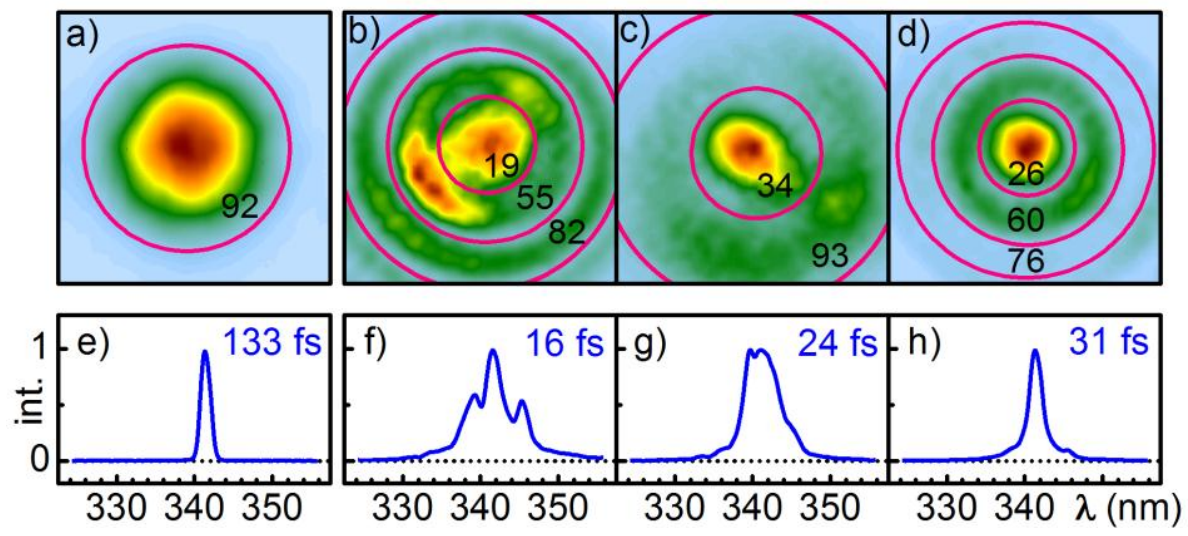

\section{Application: Collinear Two-Dimensional Spectroscopy on Pyrene}

Two-dimensional electronic spectroscopy can be regarded as an excitation frequency resolved pump-probe experiment. The measurable excitation window is determined by the spectral bandwidth of the excitation. Pulses with a spectral width large enough to cover the transitions to a multitude of vibronic or even electronic states are therefore an essential requirement. In contrast to $2 \mathrm{D}-\mathrm{Vis}[32,33]$, where a bandwidth up to $150 \mathrm{~nm}$ can be reached, experimental realizations in the UV are so far limited to about $5 \mathrm{~nm}$ [34-36].

We therefore introduce SPM broadened pulses in our collinear 2D-UV experiment. The central wavelength is set to $312 \mathrm{~nm}$ to excited the $\mathrm{S}_{2}{ }^{1} \mathrm{~B}_{2 \mathrm{u}} \leftarrow \mathrm{S}_{0}$ electronic transition of the test molecule pyrene. We use the same experimental parameters as determined above $(1 \mathrm{~mm} \mathrm{CaF} 2$ and $2.5 \mu \mathrm{J}$ input energy) and achieve a FWHM bandwidth of $13 \mathrm{~nm}$. This pulse spectrum is large enough to cover two vibronic bands of the absorption spectrum (see Figure $8 \mathrm{c}$, black line).

The 2D experiment is carried out in a pulse shaper assisted pump-probe geometry, as described in refs. [34,37]. After the SPM stage the UV beam is compressed with a UV prism compressor and then split into a pump and a probe beam. The pump passes through the AOPDF, which is used to resolve the excitation frequency by generating phase locked double pulses and scanning their interpulse delay. Additionally the shaper compensates for the higher order chirp. Both pump and probe are focused inside a $120 \mu \mathrm{m}$ thick flow cell as described in ref. [38]. The energy of the pump pulse in the cell is $63 \mathrm{~nJ}$ at a duration of the sub-pulses of $22 \mathrm{fs}$ and the probe energy is about $2 \mathrm{~nJ}$. After the sample cell the probe is spectrally resolved in a prism based CCD spectrometer. To increase the signal-to-noise ratio a fraction of the probe beam is used as a reference in a second prism based spectrometer [39]. 
Figure 8. SPM broadened UV pulses in collinear two-dimensional spectroscopy as pump and probe pulses. The absorptive signal resolves the pump-probe frequency correlation of the ground-state bleach of Pyrene in methanol for a population delay of $100 \mathrm{ps}$ (a). The spectral intensity of the pulses (blue hashed areas) and the absorption spectrum of Pyrene (black line) are shown in the right (b) and lower side panel (c).

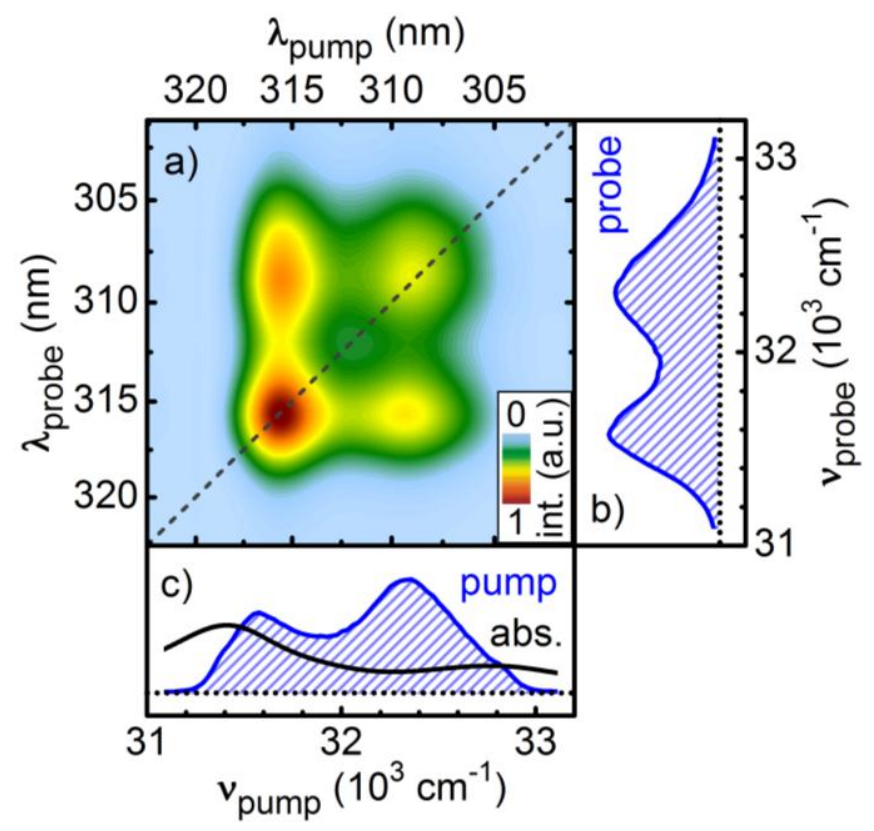

In Figure 8a the absorptive part of the 2D-UV measurement of pyrene dissolved in methanol is shown for a pump-probe delay of $100 \mathrm{ps}$. All signals in the 2D spectrum are positive and are assigned to ground state bleach contributions of pyrene. The two diagonal peaks correspond to the two vibronic bands in the absorption spectrum. In addition, due to the large spectral bandwidth of the pump pulses, the off-diagonal peaks describing the correlation between the vibronic bands are clearly resolved for the first time.

\section{Summary and Perspectives}

In this work we have shown that SPM in bulk materials can be readily used to broaden the spectrum of $\mu \mathrm{J}$ femtosecond $\mathrm{UV}$ pulses and to reduce the pulse length. The broadening stage is most conveniently incorporated into an optical setup by using two lenses or spherical mirrors with a slight off-axis geometry and a crystal plate and thus relies only on components present in most ultrafast spectroscopy laboratories. There is no need for a specially designed fiber or meter long gas cells.

By focusing the UV beam into the crystal the high pulse intensity leads to self-phase modulation and self-focusing. In contrast to techniques using gas filled cells [10] where self-focusing induced filamentation is a prerequisite for broadening, we find that the self-focus should be kept just outside the crystal, about $25 \%$ of the crystal thickness. This leads to broadening without serious beam distortions and to no beam break-up. Under this condition the pulse energy is nearly conserved as multi-photon absorption is minimized. It allows a maximum achievable broadening of up to a factor of three. Thin crystals with high nonlinearity in conjunction with reasonably tight focusing are found optimal as this keeps the negative influence of the strong group delay dispersion in the UV and the 
associated pulse lengthening to a minimum. A slight up chirp of the input pulse helps to enhance the broadening. Experimentally we find that a few millimeter thick $\mathrm{CaF}_{2}$ crystal delivers the best results in terms of broadening and transmission efficiency. Once the pulse energy and length, and the crystal thickness is known, the beam size at the plate can be determined from the well known formula for the self-focal length.

Temporal compression of the broadened pulses is done with a simple prism compressor for the regime down to about 50 fs. A compression by a factor of two to three is demonstrated. For even shorter pulses an AOPDF (DAZZLER) is added for the compensation of higher order chirp components. For pulses at $312 \mathrm{~nm}$ and $273 \mathrm{~nm}$ this allowed for a near Fourier limited compression down to 15 fs and 20 fs. If the pulses do not have to be perfectly compressed, even larger spectral broadening can be achieved.

The beam profile is quite acceptable for spectroscopic applications. The use of a circular aperture to select the central part of beam is often helpful. Still many tens of percent of the pulse energy can be utilized with the resulting new-Gaussian transversal profile.

We expect that SPM in bulk material can be employed for a large range of energies as the focusing conditions can be adapted for pulses less than a $\mu \mathrm{J}$ to tens of $\mu \mathrm{J}$. Although here we only investigated the broadening of 120 and 25 fs input pulses, we envision that pulses at various pulse length can be broadened and compressed. The method likely will be best suited to situations where just an extra factor around two is needed for a successful experiment. This is, to our own experience, quite often the case.

\section{Acknowledgments}

We thank Thomas Ganz for valuable discussions and suggestions. This work was supported by the Austrian Science Fund within the framework of the Special Research Program F16 (Advanced Light Sources), by the SFB749 and by the DFG-Cluster of Excellence: Munich-Centre for Advanced Photonics.

\section{References}

1. Krebs, N.; Probst, R.A.; Riedle, E. Sub-20 fs pulses shaped directly in the UV by an acousto-optic programmable dispersive filter. Opt. Express 2010, 18, 6164-6171.

2. Homann, C.; Lang, P.; Riedle, E. Generation of 30 fs pulses tunable from 189 to $240 \mathrm{~nm}$ with an all-solid-state setup. J. Opt. Soc. Am. B 2012, 29, 2765-2769.

3. Baum, P.; Lochbrunner, S.; Riedle, E. Generation of tunable 7-fs ultraviolet pulses: achromatic phase matching and chirp management. Appl. Phys. B 2004, 79, 1027-1032.

4. Zhao, B.; Jiang, Y.; Sueda, K.; Miyanaga, N.; Kobayashi, T. Sub-15fs ultraviolet pulses generated by achromatic phase-matching sum-frequency mixing. Opt. Express 2009, 17, 17711-17714.

5. Kida, Y.; Liu, J.; Kobayashi, T. Single 10-fs deep-ultraviolet pulses generated by broadband four-wave mixing and high-order dispersion compensation. Appl. Phys. B 2011, 105, 675-679.

6. Backus, S.; Peatross, J.; Zeek, Z.; Rundquist, A.; Taft, G.; Murnane, M.M.; Kapteyn, H.C. 16-fs, $1-\mu \mathrm{J}$ ultraviolet pulses generated by third-harmonic conversion in air. Opt. Lett. 1996, 21, 665-667.

7. Reiter, F.; Graf, U.; Schultze, M.; Schweinberger, W.; Schröder, H.; Karpowicz, N.; Azzeer, A.M.; Kienberger, R.; Krausz, F.; Goulielmakis, E. Generation of sub-3 fs pulses in the deep ultraviolet. Opt. Lett. 2010, 35, 2248-2250. 
8. Noack, F.; Steinkellner, O.; Tzankov, P.; Ritze, H.-H.; Herrmann, J.; Kida, Y. Generation of sub30 fs ultraviolet Raman induced phase modulation in nitrogen. Opt. Express 2005, 13, 2467-2474.

9. Durfee III, C.G.; Backus, S.; Kapteyn, H.C.; Murnane, M.M. Intense 8-fs pulse generation in the deep ultraviolet. Opt. Lett. 1999, 24, 697-699.

10. Ghotbi, M.; Trabs, P.; Beutler, M. Generation of high-energy, sub-20-fs pulses in the deep ultraviolet by using spectral broadening during filamentation in argon. Opt. Lett. 2011, 36, 463-465.

11. Nagy, T.; Simon, P. Generation of 200- $\mu$ J, sub-25-fs deep-UV pulses using noble-gas-filled hollow fiber. Opt. Lett. 2009, 34, 2300-2302.

12. Ganz, T.; Pervak, V.; Apolonski, A.; Baum, P. $16 \mathrm{fs}, 350 \mathrm{~nJ}$ pulses at $5 \mathrm{MHz}$ repetition rate delivered by chirped pulse compression in fibers. Opt. Lett. 2011, 36, 1107-1109.

13. Alfano, R.R.; Shapiro, S.L. Observation of self-phase modulation and small-scale filaments in crystals and glasses. Phys. Rev. Lett. 1970, 24, 592-594.

14. Hata, K.; Watanabe, M.; Watanabe, S. Nonlinear Processes in UV Optical Materials. Appl. Phys. B 1990, 50, 55-59.

15. Spanner, M.; Ivanov, M.Y.; Kalosha, V.; Hermann, J.; Wiersma, D.A.; Pshenichnikov, M. Tunable optimal compression of ultrabroadband pulses by cross-phase modulation. Opt. Lett. 2003, 28, 749-751.

16. Petrov, V.; Rudolph, W.; Wilhelmi, B. Compression of high-energy femtosecond light pulses by self-phase modulation in bulk media. J. Mod. Opt. 1989, 36, 587-595.

17. Rolland, C.; Corkum, P.B. Compression of high-power optical pulses. J. Opt. Soc. Am. B 1988, 5, 641-647.

18. Tzortzakis, S.; Lamouroux, B.; Chiron, A.; Franco, M.; Prade, B.; Mysyrowicz, A.; Moustaizis, S.D. Nonlinear propagation of subpicosecond ultraviolet laser pulses in air. Opt. Lett. 2000, 25, 1270-1272.

19. Kim, Y.P.; Hutchinson, M.H.R. Intensity-Induced Nonlinear Effects in UV Window Materials. Appl. Phys. B 1989, 49, 469-478.

20. Diels, J.-C.; Rudolph, W. Ultrashort Laser Pulse Phenomena; Academic Press: London, UK, 1995.

21. Fibich, G.; Gaeta, A.L. Critical power for self-focusing in bulk media and in hollow waveguides. Opt. Lett. 2000, 25, 335-337.

22. Schmidt, B.; Hacker, M.; Stobrawa, G.; Feurer, T. LAB2-A Virtual Femtosecond Laser Lab. Available online: http://www.lab2.de (accessed on 1 September 2012).

23. DeSalvo, R.; Said, A.A.; Hagan, D.J.; Van Stryland, E.W.; Sheik-Bahae, M. Infrared to Ultraviolet Measurements of Two-Photon Absorption an $n_{2}$ in Wide Bandgap Solids. IEEE J. Quantum Electron. 1996, 32, 1324-1333.

24. Kozma, I.Z.; Baum, P.; Lochbrunner, S.; Riedle, E. Widely tunable sub-30 fs ultraviolet pulses by chirped sum frequency mixing. Opt. Express 2003, 11, 3110-3115.

25. Coudreau, S.; Kaplan, D.; Tournois, P. Ultraviolet acousto-optic programmable dispersive filter laser pulse shaping in KDP. Opt. Lett. 2006, 31, 1899-1901.

26. Homann, C.; Krebs, N.; Riedle, E. Convenient pulse length measurement of sub-20-fs pulses down to the deep UV via two-photon absorption in bulk material. Appl. Phys. B 2011, 104, 783-791.

27. Baum, P.; Lochbrunner, S.; Riedle, E. Zero-additional-phase SPIDER: full characterization of visible and sub-20-fs ultraviolet pulses. Opt. Lett. 2004, 29, 210-212. 
28. Oberthaler, M.; Höpfel, R.A. Special narrowing of ultrashort laser pulses by self-phase modulation in optical fibers. Appl. Phy. Lett. 1993, 63, 1017-1019.

29. Yariv, A. Quantum Electronics, 3rd ed.; John Wiley \& Sons: New York, NY, USA, 1988; p. 487.

30. Nascimento, C.M.; Alencar, M.A.R.C.; Chávez-Cerda, S.; da Silva, M.G.A.; Meneghetti, M.R.; Hickmann, J.M. Experimental demonstration of novel effects on the far-field diffraction patterns of a Gaussian beam in a Kerr medium. J. Opt. A: Pure Appl. Opt. 2006, 8, 947-951.

31. Garcia Ramirez, E.V.; Arroyo Carrasco, M.L.; Mendez Otero, M.M.; ChavezCerda, S.; Iturbe Castillo, M.D. Far field intensity distributions due to spatial selfphase modulation of a Gaussian beam by a thin nonlocal nonlinear media. Opt. Express 2010, 18, 22067-22079.

32. Christensson, N.; Milota, F.; Nemeth, A.; Pugliesi, I.; Riedle, E.; Sperling, J.; Pullerits, T.; Kauffmann, H.F.; Hauer, J. Electronic Double-Quantum Coherences and Their Impact on Ultrafast Spectroscopy: The Example of $\beta$-Carotene. J. Phys. Chem. Lett. 2010, 1, 3366-3370.

33. Bixner, O.; Lukeš, V.; Maňcal, T.; Hauer, J.; Milota, F.; Fischer, M.; Pugliesi, I.; Bradler, M.; Schmid, W.; Riedle, E.; et al. Ultrafast photo-induced charge transfer unveiled by two-dimensional electronic spectroscopy. J. Chem. Phys. 2012, 136, 204503.

34. Tseng, C.-H.; Matsika, S.; Weinacht, T.C. Two-Dimensional Ultrafast Fourier Transform Spectroscopy in the Deep Ultraviolet. Opt. Express 2009, 17, 18788-18793.

35. Selig, U.; Schleussner, C.-F.; Foerster, M.; Langhojer, F.; Nuernberger, P.; Brixner, T. Coherent two-dimensional ultraviolet spectroscopy in fully noncollinear geometry. Opt. Lett. 2011, 35, 4178-4180.

36. West, B.A.; Womick, J.M.; Moran, A.M. Probing Ultrafast Dynamics in Adenine With Mid-UV Four-Wave Mixing Spectroscopies. J. Phys. Chem. A 2011, 115, 8630-8637.

37. Shim, S.-H.; Zanni, M.T. How to turn your pump-probe instrument into a multidimensional spectrometer: 2D IR and Vis spectroscopies via pulse shaping. Phys. Chem. Chem. Phys. 2009, $11,748-761$.

38. Megerle, U.; Pugliesi, I.; Schriever, C.; Sailer, C.F.; Riedle, E. Sub-50 fs broadband absorption spectroscopy with tunable excitation: putting the analysis of ultrafast molecular dynamics on solid ground. Appl. Phys. B 2009, 96, 215-231.

39. Dobryakov, A.L.; Kovalenko, S.A.; Weigel, A.; Pérez-Lustres, J.L.; Lange, J.; Müller, A.; Ernsting, N.P. Femtosecond pump/supercontinuum-probe spectroscopy: Optimized setup and signal analysis for sinlge shot spectral referencing. Rev. Sci. Instrum. 2010, 81, 113106.

(C) 2013 by the authors; licensee MDPI, Basel, Switzerland. This article is an open access article distributed under the terms and conditions of the Creative Commons Attribution license (http://creativecommons.org/licenses/by/3.0/). 